\title{
Effects of Gender and Obesity on Daytime Sleepiness in Obstructive Sleep Apnoea Patients
}

\author{
Sushil Kumar Sah ${ }^{1, *}$, Hua Zhang Tan ${ }^{1}$, Sun Yu ${ }^{2}$, Jin Feng ${ }^{1}$, Yong Hong Zhang ${ }^{1}$ and Anil Kumar \\ Shah ${ }^{3}$ \\ 1 Department of Otorhinolaryngology, Xiangyang No.1 Peoples Hospital, Hubei University of Medicine, \\ Shiyan, Hubei 442000, China \\ 2 Department of Otorhinolaryngology, Wuhan Union Hospital, Wuhan, China \\ * Correspondence: sushilsah90@yahoo.com
}

\begin{abstract}
Objective: Identify factors associated with excessive day time sleepiness (EDS) in individuals with obstructive sleep apnea (OSA), and analyze the effects that obesity and gender have on excessive daytime sleepiness in such individuals. Methods: A total of 160 people were selected for this study. All the people have completed a clinical evaluation, and whose apneahypopnea index $(\mathrm{AHI})>10$ events/hour of sleep on polysomnography were included in the study from the Department of otolaryngology. Results: The Mean age was $43.87 \pm 11.34$ years, mean EDS score was $14.09 \pm 4.91$, and mean AHI was $43.88 \pm 20.66$ events/hour of sleep. Male presented lower mean age, higher EDS scores, and more time in apnoea, whereas females presented with higher mean age, lower EDS scores, and less time in sleep apnea. The EDS score showed best correlation with duration of apnoea $(\mathrm{r}=0.448 ; \mathrm{p}<0.01)$, peripheral oxygen saturation $\left(\mathrm{SpO}_{2} ; \mathrm{r}=-0.458 ; \mathrm{p}<0.01\right)$ and AHI $(\mathrm{r}=0.484 ; \mathrm{p}<0.01)$. The mean body mass index (BMI) was $27.41 \pm 3.86 \mathrm{~kg} / \mathrm{m}^{2}$. Normal, Overweight, obese, and morbidly obese were observed in $20 \%, 61 \%, 18 \%$, and $0.6 \%$ of cases, respectively. Severity of the disease best correlated with BMI $(\mathrm{r}=0.421 ; \mathrm{p}<0.01)$. Conclusions: OSA is predominant in males (M/F 5:1), and obese population. Females diagnosed with OSA have higher mean age. However, EDS scores and time spent in sleep apnoea is lower in females. Higher BMI is associated with EDS, irrespective of gender.
\end{abstract}

Keywords: sleep apnoea; obstructive sleep apnoea; polysomnography; excessive day time sleepiness; obesity

\section{Introduction}

Excessive day time sleepiness (EDS), or hypersomnolence is today's rising concern in many societies. It is associated with obesity and is common in patients with obstructive sleep apnoea (OSA). Obesity and overweight prevalence have grown around the world, according to the World Health Organization says the worldwide percentage has tripled since 1975. The world's most populous country, China, the rate of overweight and obesity increases is even higher. [1] Obstructive sleep apnoea (OSA) is a disorder characterized by repeated events of impaired ventilation during sleep, caused by obstruction of the upper airways. [2] These events can be total (apnoeas) or partial (hypopneas) and are often associated with episodes of decreased arterial blood oxygenation level (i.e., desaturations) and brief arousals from sleep. The term OSA syndrome (OSAS) is often used to denote symptomatic OSA, e.g., OSA with daytime sleepiness. The diagnosis is made based on a polysomnographic recording of physiological signals related to breathing (e.g., nasal airflow, chest, and abdominal movement, heart rate, and blood oxygen saturation) during sleep. Sleep can be assessed by Electrophysiological technology such as direct polysomnography (PSG), consisting of electroencephalography (EEG), surface electromyography (EMG), and electrooculography (EOG), heart rate, or actigraphy or sleep diary. [3-4] OSA severity is often based on the number of apneas and hypopneas per hour of sleep (the Apnoea Hypopnea Index, AHI) and the number of oxygen 
desaturation events per hour of sleep (the Oxygen Desaturation Index, ODI). OSA has been associated with hypertension, cardiovascular disease, [5] traffic accidents, [6] obesity, and hyperglycemia/type II diabetes mellitus. [7] The reasons are not completely clear, although several factors have been identified that are associated with treatment adherence. The most commonly reported symptom of OSAS is daytime sleepiness, which is often measured using the Epworth Sleepiness Scale. [8] Both OSA and EDS are a public health problem. It not only leads to cognitive impairment but also reduced reflexes, resulting in an increased risk of traffic accidents and other mishaps. Also, EDS can have significant repercussions for work, social life, and quality of life. [9] The objective of this study was to evaluate the impact of gender and obesity in patients with OSA.

\section{Methodology}

\subsection{Equipment}

Polysomnographic tests were performed using the standard method, with an electroencephalogram, electrooculogram (left eye and right eye), electromyogram (chin and anterior tibia), and electrocardiogram. All of the equipment employed was manufactured by Beijing Xin Yang Sheng poly Technology (China). Airflow was measured using a nasal cannula connected to a pressure transducer, and peripheral oxygen saturation $\left(\mathrm{SpO}_{2}\right)$ was measured using a pulse oximeter (China).

\subsection{Study Design and Sample Population}

All of the patients were examined retrospectively. Patients with OSA of the 4th affiliated hospital of the Hubei University of Medicine and the sample population were the patients who were admitted into the 4th Affiliated Hospital of Hubei University of medicine from January 2016 to July 2017.

\subsection{Ethical consideration and approval of informed consent}

By using a pre-established protocol patient were included in the study, the patients have explained the purpose of the study and Once the verbal consent was obtained with the presence of a Nurse especially trained in Otolaryngology, a written form has given both in English and Chinese language to get the written informed consents.

\subsection{Study population}

A total of 160 patients diagnosed with OSA, who had been submitted polysomnography, were eligible for inclusion in the study. Of those, 160 patients were selected and subsequently evaluated over a one and half year's period. Patients were selected based on the following criteria: clinical chart data being complete; being over 16 years of age; and having received a diagnosis of OSA confirmed through polysomnography, apnoea-hypopnea index (AHI) being $>10$ events/hour of sleep. Clinical data were obtained through history and physical examination. Upon history, conducted with the patient and their family member, questions were asked regarding snoring, sleep apnea, and daytime sleepiness, including the patient history of traffic accidents. We investigated EDS using the criteria proposed by Johns MW. [10] The patients were asked how often they fell dozing in different situations, such as sitting and reading, watching television, meeting or theatre, traveling as a passenger, lying down in the afternoon, sitting and talking, sitting quietly after lunch, in a car while stopped in traffic. (Possible responses: never; slight; mild; moderate; and high). A score was attributed to each answer, ranging from 0 to 3 , in which 0 corresponded to never and 3 corresponded to high. Therefore, the overall EDS score ranged from 0 to 24. [10]

The body mass index (BMI) for each patient was obtained by dividing weight in kilograms by height in meters squared. Patients were categorized by weight status based on their BMI: normal (between 20 and $24.9 \mathrm{~kg} / \mathrm{m}^{2}$ ), overweight (BMI between 25 and $29.9 \mathrm{~kg} / \mathrm{m}^{2}$ ), obese (BMI between 30 and $\left.39.9 \mathrm{~kg} / \mathrm{m}^{2}\right)$, or morbidly obese (BMI $\left.\geq 40 \mathrm{~kg} / \mathrm{m}^{2}\right)$. Sleep stages were classified according to the criteria established by U. Rajendra Acharya, Yildirim O, and Baloglu UB. [10] Apnoea was defined as a reduction in airflow to $\leq 10 \%$ of the baseline value for 10 seconds or more, and hypopnea was 
defined as reductions in airflow of $\geq 50 \%$ accompanied by awakening and a $\geq 4 \%$ drop in $\mathrm{SpO}_{2}$. The AHI was calculated by dividing the total number of apnoea-hypopnea episodes by the number of hours of sleep. Based on AHI, OSA was classified as Mild (<15), moderate (15-30), or severe (>30). [1112]

\subsection{Data analysis}

Data were stored and analyzed using the program Statistical Package for Social Sciences, version 22.0 (SPSS Inc, Chicago, IL, USA). Results are expressed as many cases, percentages, mean and standard deviation, or standard error. To analyze the correlations among variables with normal distribution, Pearson's linear correlation test was used, whereas Spearman's correlation coefficient was used for variables with non-normal distribution. All of the tests were two-tailed. Values of $\mathrm{p}<$ 0.05 were considered statistically significant.

\section{Results}

A total of 160 patients with OSA, 134 were male and 26 were female. The mean age was $43.87 \pm 11.34$ years, varying from 16 to 77 years. Mode age was in between 41 to 50 years in both males and females. Clinical and polysomnographic data are shown, by gender (see Table 1). There was no difference in terms of reported snoring or apnoea with gender (chi-square; $p>0.05$ ). Significant differences were observed between males and females in relation to the following: mean age (41.64 \pm 9.74 years vs. $46.54 \pm 12.30$ years; $\mathrm{p}=0.031)$; mean EDS score $(14.17 \pm 4.95$ vs. $13.37 \pm 4.92 ; \mathrm{p}=0.000)$; and meantime spent in sleep apnoea $(167.96 \pm 97.61 \mathrm{~min}$ vs. $124.25 \pm 83.16 \mathrm{~min} ; \mathrm{p}=0.041)$. There were no statistically significant differences between genders in terms of the remaining clinical and polysomnographic variables. The mean EDS score for the sample as a whole was $14.09 \pm 4.91$. We found that EDS score correlated positively and significantly with the following variables: BMI; total sleep time; sleep efficiency; a percentage of total sleep time spent in REM sleep; the total number of minutes spent in sleep apnoea; and percentage of total sleep time spent in apnoea statistically significant. Although negative, correlations were observed between the EDS score and the following variables: age; sleep latency; mean $\mathrm{SpO}_{2}$; (see Table 2).

The mean BMI in the sample as a whole was $27.41 \pm 3.86$. Of the 160 patients evaluated, $128(80 \%)$ presented a BMI $\geq 25 \mathrm{~kg} / \mathrm{m}^{2}$. There were 98 patients $(61 \%)$ who were classified as overweight, $29(18 \%)$ who were classified as obese, and $1(0.6 \%)$ who were classified as morbidly obese. Figure 2 . Shows the AHI, total sleep time spent in apnoea in percentage, and the minimum $\mathrm{SpO}_{2}$ after the categorization of the patients by BMI. There were simultaneous increases in AHI and in the percentage of time spent in sleep apnea as increases in the degree of obesity. In obese patients both the AHI and percentage of time spent in sleep apnoea is almost similar to the lowest minimum $\mathrm{SpO}_{2} . \mathrm{SpO}_{2}$ is in all the variables normal, overweight, and obese patients have not much difference. We found that BMI correlated more strongly with the EDS score $(r=0.701 ; p<0.001)$ than with any other clinical variable. (see figure 1b) The polysomnographic variables with which BMI correlated most strongly were AHI ( $r=0421 . p$ $<0.001)$, minimum $\mathrm{SpO}_{2}(\mathrm{r}=-0.370 ; \mathrm{p}<0.001)$ and sleep efficiency $(\mathrm{r}=0.214 ; \mathrm{p}<0.001)$.

The OSA was mild (AHI < 15) in 10 patients, moderate (AHI between 15 and 30) in 36 patients, and severe (AHI > 30) in 113 patients. The mean EDS score was $12.72 \pm 1.77$ in the group with mild disease, $20.84 \pm 3.96$ in the group with moderate disease, and $54.42 \pm 15.82$ in the group with severe disease $(p<0.01)$. There were no differences among the groups in terms of age $(p=0.37)$. There were significant differences among the three OSA severity groups in terms of mean BMI (mild OSA: 27.40 $\pm 3.20 \mathrm{~kg} / \mathrm{m}^{2}$; moderate OSA: $32.01 \pm 1.25 \mathrm{~kg} / \mathrm{m}^{2}$; and severe OSA: $48.25 \pm 9.68 \mathrm{~kg} / \mathrm{m}^{2}$. We also found AHI to correlate better with BMI and EDS scores than with any other clinical variables (Figure $3 a$ ). As for the polysomnographic variables. AHI best correlate with body mass index ( $r=0.421 ; \mathrm{p}<0.001)$. There is a negative correlation found with minimum $\mathrm{SpO}_{2}(\mathrm{r}=-0.458 ; \mathrm{p}<0.00)$. We also found AHI correlates better with BMI and EDS scores than with any other clinical variables (Figure $3 a$ and $b$ ). As for the polysomnographic variables. AHI best correlate with body mass index $(r=0.421 ; \mathrm{p}<0.001)$.

\section{Discussion}


Sleep disorders have been always a disturbing public health issue that affects the quality of life [13]. Therefore, we conducted a cross-sectional study involving patients with OSAHS. Gender-related characteristics were compared and the effects of obesity on the disease were analyzed. Our study identified the predominance of the disease in males. The severity of the disease was similar in both genders. Females presented higher mean age, lower EDS scores, and less time spent in sleep apnoea. Among the patients in our study, the mean age at diagnosis was similar to that reported in other studies. [14-15] Cases of OSA can occur from adolescence to old age. The fact that the incidence of the disease peaks the same decade both in females and males but in males it begins the first half of the decade whereas in the female latter half of the decade, as found in this and other studies. In females, it has been attributed to the effect of hormonal protection of premenopausal females against OSA. [16]

A 5:1 male/female ratio was found in our sample of patients, whereas one of the most often cited articles on the prevalence of OSA in the literature reported a 2:1 ratio. [17-18] The lower proportion of females in the present study can be explained by the different presentation of the disease in females and by the fact that diagnosing OSA in females continues to be difficult. [16] Also, the females in our study presented less EDS and remained less time in sleep apnoea, although disease severity in females was comparable to that observed in males. Various factors associated with EDS, such as obesity, alterations in sleep architecture, were identified in our study. However, the correlations between the EDS score and the remaining variables, although significant and ranged from weak to moderate, which suggests that other factors can be associated with EDS. Factors such as fragmented sleep due to respiratory events have previously been related to EDS. [19]

EDS is one of the major consequences of OSA. EDS has a greater frequency of traffic accidents due to drivers falling asleep at the wheel. [20-22] Of the patients studied, $16 \%$ to $20 \%$ reported having accidents or near accidents due to falling asleep at the wheel. This is slightly lesser than the $29 \%$ previously reported. [21] Work accident was found to be nearly double in workers with OSA (OR = $2.18 ; 95 \% \mathrm{CI}=1.53-3.10)$. Occupational driving was associated with a higher effect size. [22-24] It has been shown that a higher frequency of traffic accidents is also associated with factors other than OSA: the use of alcohol, chronic sleep deprivation, and narcolepsy, restless legs syndrome, and periodic limb movements, circadian rhythm sleep disorders, and the use of medication. [23] Of the 160 patients, $80.0 \%$ were considered overweight or obese. In male BMI $>25$ is $85 \%$ whereas, in females, it is only $66 \%$. In addition to being associated with OSA severity, obesity is one of the risk factors for cardiovascular diseases. [25] OSA and hypertension coexist, the prevalence of the OSA in hypertensive people has markedly increased. [26] In our study, $51 \%$ of patients were found hypertensive.

We consider that our sample size was adequate to identify the effects of gender and obesity on excessive day time sleepiness in OSA patients. There were few major limitations of the present study such as the lack of a control group composed of gender- and age-matched individuals, lack of a wellequipped polysomnographic machine. Another limitation, in terms of our ability to evaluate differences related to gender and disease severity, was the heterogeneity of the sample; only 26 patients $(16.25 \%)$ were female, 134 were male $(83.75 \%)$, and $113(70.62 \%)$ presented severe OSA.

\section{Conclusion}

OSA is considered as a male predominant (M/F 5:1), and common in the obese population. Females diagnosed with OSA have higher mean age. However, EDS scores and time spent in sleep apnoea are lower in females. Higher BMI is associated with EDS, irrespective of gender. Other elements such as fragmented sleep, minimum $\mathrm{SpO}_{2}$ levels, and degree of obesity were associated with EDS.

Conflicts of Interest: None to declare

Author Contributions: SKS: concept and protocol development, review plan, data collection, data analysis, manuscript writing, statistical analysis, review, and editing of the manuscript. ZHT, JF, SY: review, and manuscript supervision. YHZ: data and consent collection. AKS: editing of the manuscript. 
Declaration: All the authors approved to publish this work.

Funding: Not applicable.

Acknowledgments: Thanks to the Department of Otolaryngology Xiangyang No.1 People Hospital, and the Hubei University of Medicine, Shiyan, China for their support and help throughout this project.

\section{References}

[1] L Zhang, Z Wang, X Wang, Z Chen, L Shao, et al. Prevalence of overweight and obesity in China: results from a cross-sectional study of 441 thousand adults, 2012-2015, doi.org/10.1016/j.orcp.2020.02.005

[2] Ito E et al. The International Classification of Sleep Disorders, third edition. American Academy of Sleep Medicine. Includes bibliographies and index]. Nihon Rinsho. (2015)

[3] Syeda A, Karim MR. The mean age of petit mal epilepsy. J Pediatr Neurosci. 2016 Apr 1; 11(2):112-114. doi:10.4103/1817-1745.187627

[4] Hsu YS, Chen TY, Wu D, Lin CM, Juang JN, Liu WT. J Clin, Screening of Obstructive Sleep Apnoea in Snoring Patients Using a Patch-Type Device with Electrocardiogram and 3-Axis Accelerometer. Sleep Med. 2020 Apr 8. doi: 10.5664/jcsm.8462

[5] Ben Halima A, Aouadi S, Bejjar D, Laroussi L, Boukhris M, Gharbi L, Addad F, Kachboura S. Hypertension and Atrial Fibrillation: What is the prevalence of obstructive sleep apnoea syndrome? Tunis Med. 2018 Mar;96(3):187-192

[6] Lichtblau M, Bratton D, Giroud P, Weiler T, Bloch KE, Brack T. Risk of Sleepiness-Related Accidents in Switzerland: Results of an Online Sleep Apnea Risk Questionnaire and Awareness Campaigns. Front Med (Lausanne). 2017 Apr 12; 4:34. doi: 10.3389/fmed.2017.00034

[7] Mokhlesi B, Temple KA, Tjaden AH, Edelstein SL, Nadeau KJ, Hannon TS, Manchanda S, Sam S, Barengolts E, Utzschneider KM, Ehrmann DA, Van Cauter E. The association of sleep disturbances with glycemia and obesity in youth at risk for or with recently diagnosed type 2 diabetes. RISE Consortium. Pediatr Diabetes. 2019 Dec; 20(8):1056-1063. doi: 10.1111/pedi.12917

[8] Omobomi O, Batool-Anwar S, Quan SF. Clinical and Polysomnographic Correlates of Subjective Sleepiness in Mild Obstructive Sleep Apnea. Sleep Vigil. 2019 Dec; 3(2):131-138. doi: 10.1007/s41782-019-00068-2

[9] Léger D, Stepnowsky C. The economic and societal burden of excessive daytime sleepiness in patients with obstructive sleep apnoea. Sleep Med Rev. 2020 Feb 17; 51:101275. doi: 10.1016/j.smrv.2020.101275

[10] Johns, MW (1991): A New Method for Measuring Daytime Sleepiness: the Epworth Sleepiness Scale. Sleep 14(6):540-545.

[11] Warren R. Ruehland, Peter D. Rochford, et al, The New Aasm Criteria for Scoring Hypopneas: Impact on the Apnea Hypopnea Index, doi.org/10.5665/sleep/32.2.150

[12] Ruehland WR, Rochford PD, et al, The new AASM criteria for scoring hypopneas: impact on the apnea hypopnea index, 2009 Sleep. 32 (2): doi:10.1093/sleep/32.2.150

[13] Liu GJ, Karim MR, Xu LL, Wang SL, Yang C, Ding L, Wang YF. Efficacy and tolerability of gabapentin in adults with sleep disturbance in medical illness: a systemic review and meta-analysis. Front Neurol. 2017 Jul 14; 8:316. doi:10.3389/fneur.2017.00316

[14] S Tufik, R Santos-Silva, JA Taddei, LRA Bittencourt. Obstructive sleep apnoea syndrome in the Sao Paulo epidemiologic sleep study - Sleep medicine, Volume 11, Issue 5, May 2010, doi.org/10.1016/j.sleep.2009.10.005

[15] Kapsimalis F, Kryger MH. Gender and obstructive sleep apnoea syndrome, part 2: mechanisms. Sleep. 2002; 25(5):499-506. doi.org/10.1093/sleep/25.5.497

[16] Huang T, Lin BM, Redline S, Curhan GC, Hu FB, Tworoger SS. Type of Menopause, Age at Menopause, and Risk of Developing Obstructive Sleep Apnea in Postmenopausal Women. Am J Epidemiol. 2018 Jul 1; 187(7):1370-1379. doi: 10.1093/aje/kwy011 
[17] Hashim Z, Nath A, Gupta M, Khan A, Mishra R, Srivastava S, Tripathi SK. Diagnosis of obstructive sleep apnoea in women: Is there any difference? - Experience from a tertiary care hospital in North India. J Family Med Prim Care. 2019 Oct 31; 8(10):3276-3281. doi: 10.4103/jfmpc.jfmpc_609_19

[18] Karl A. Franklin, Eva Lindberg; Obstructive sleep apnea is a common disorder in the population - a review on the epidemiology of sleep apnea J Thorac Dis. 2015 Aug; 7(8): 1311-1322. doi: 10.3978/j.issn.20721439.2015.06.11

[19] Shao C, Qi H, Lang R, Yu B, Tang Y, Zhang L, Wang X, Wang L. Clinical Features and Contributing Factors of Excessive Daytime Sleepiness in Chinese obstructive Sleep Apnoea Patients; The role of Comorbid Symptoms and Polysomnography Variables. Can Respir J. 2019 Jul 10;2019:5476372. doi: $10.1155 / 2019 / 5476372$

[20] Purtle MW, Renner CH, McCann DA, Mallen JC, Spilman SK, Sahr SM. Driving with undiagnosed obstructive sleep apnoea (OSA): High prevalence of OSA risk in drivers who experienced a motor vehicle crash. Traffic Inj Prev.2020;21(1):38-41. doi: 10.1080/15389588.2019.1709175

[21] Ebrahimi MH, Sadeghi M, Dehghani M, Niiat KS. Sleep habits and road traffic accident risk for Iranian occupational drivers. Int J Occup Med Environ Health. 2015; 28(2):305-12. doi: 10.13075/ijomeh.1896.00360

[22] Marco Túlio de Mello, Fernanda Veruska Narciso, Sergio Tufik, et al. Sleep Disorders as a Cause of Motor Vehicle Collisions. Int J Prev Medv.4 (3); 2013 Mar

[23] Sergio Garbarino, Ottavia Guglielmi, Antonio Sanna, et al. Risk of Occupational Accidents in Workers with Obstructive Sleep Apnoea: Systematic Review and Meta-analysis. Sleep. 2016 Jun 1; 39(6): 1211-1218. doi.org/10.5665/sleep.5834

[24] C Koliaki, S Liatis, Obesity and cardiovascular disease: revisiting an old relationship. A KokkinosMetabolism, Volume 92, March 2019, Elsevierdoi.org/10.1016/j.metabol.2018.10.011

[25] Kim L. Ward, David R, et al. Excessive Daytime Sleepiness Increases the Risk of Motor Vehicle Crash in Obstructive Sleep Apnoea. J Clin Sleep Med. 2013 Oct 15; 9(10): 1013-1021.

[26] T Konecny, T Kara, VK Somers - Obstructive sleep apnea and hypertension un update hypertension,2014 - Am Heart Assoc, doi.org/10.1161/HYPERTENSIONAHA.113.00613

\section{FIGURE LEGENDS}

\section{Figure 1:}

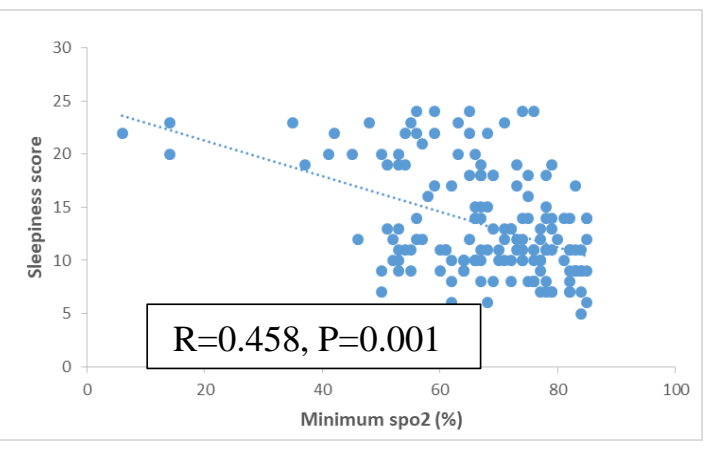

(a)

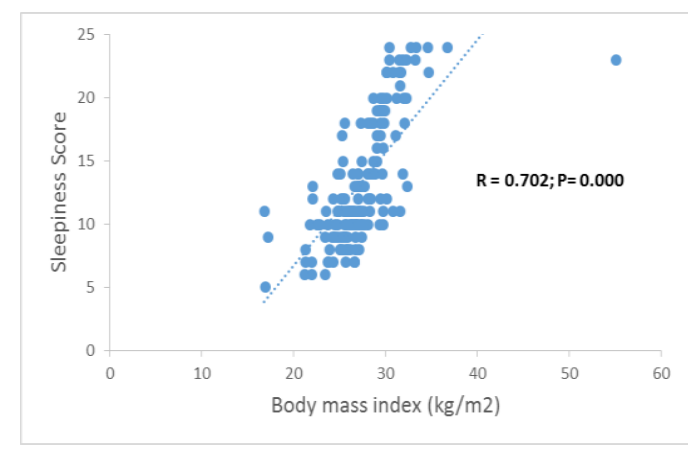

(b)

Figure 1. Correlations among the minimum peripheral oxygen saturation $\left(\mathrm{SpO}_{2}\right)(\mathrm{a})$ and excessive daytime sleepiness score, body mass index (b). 


\section{Figure 2:}

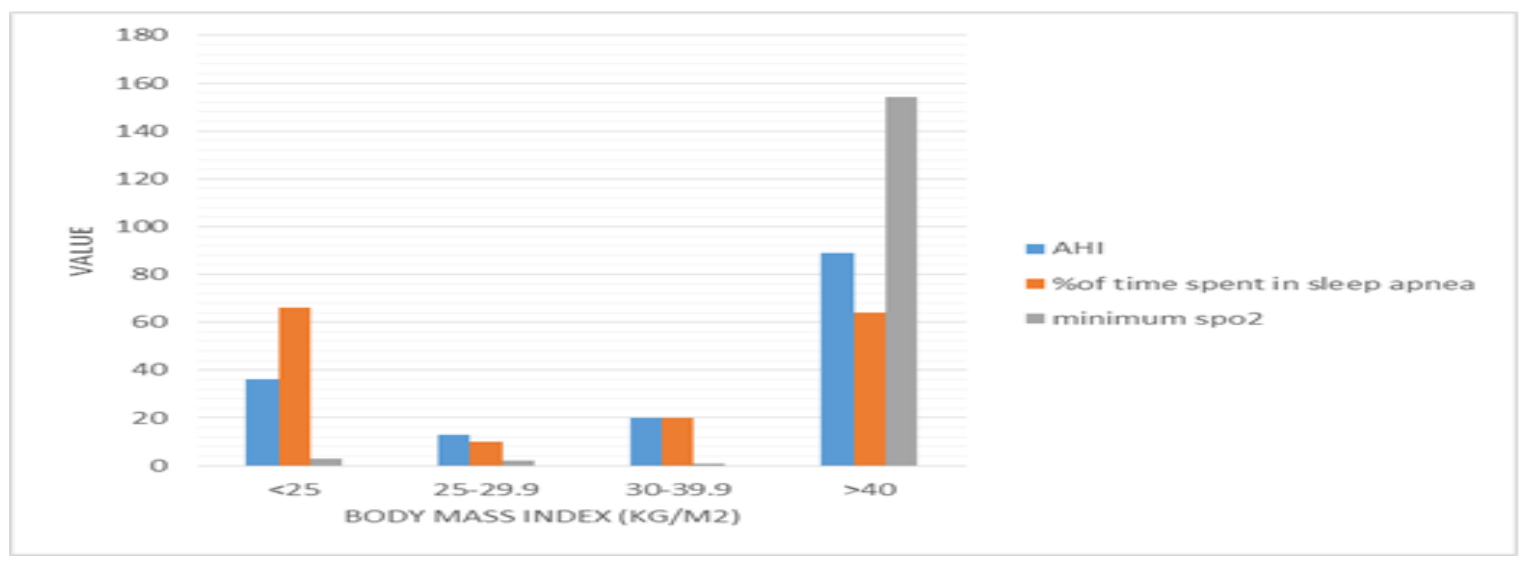

Figure 2. Mean values of an apnoea-hypopnea index (AHI), percentage of total sleep time spent in apnoea, and minimum peripheral oxygen saturation $\left(\mathrm{SpO}_{2}\right)$ in patients stratified according to the body mass index (mean \pm standard error).

\section{Figure 3:}

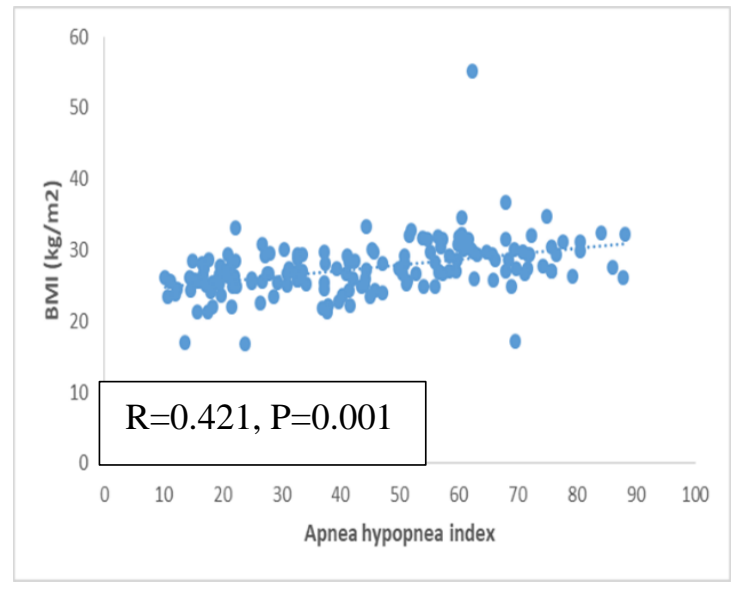

(a)

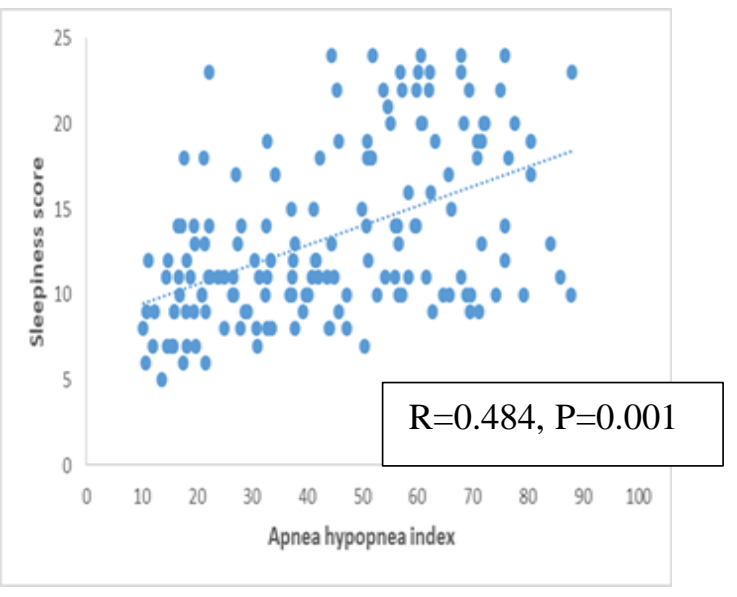

(b)

Figure 3. Correlations between apnoea-hypopnea index (AHI) and the variables body mass index (a), excessive daytime sleepiness score (b). 
TABLES:

Table 1. Clinical and polysomnographic characteristics in 160 patients with obstructive sleep apnoeahypopnea syndrome by gender.

\begin{tabular}{ccccc}
\hline Characteristics & $\begin{array}{c}\text { All } \\
\mathbf{n = 1 6 0}\end{array}$ & $\begin{array}{c}\text { Males } \\
\mathbf{n = 1 3 6}\end{array}$ & $\begin{array}{c}\text { Females } \\
\mathbf{n = 2 4}\end{array}$ & $\begin{array}{c}\mathbf{P} \\
\text { value }\end{array}$ \\
\hline Age (years) & $43.87 \pm 11.34$ & $41.64 \pm 9.74$ & $46.54 \pm 12.30$ & 0.031 \\
BMI $\left(\mathrm{kg} / \mathrm{m}^{2}\right)$ & $27.41 \pm 3.86$ & $27.57 \pm 4.03$ & $26.55 \pm 3.08$ & 0.239 \\
Symptoms (years) & $6.9 \pm 3.23$ & $6.34 \pm 2.62$ & $7.5 \pm 2.75$ & 0.49 \\
Total sleep time (min) & $430.52 \pm 60.49$ & $427.13 \pm 54.97$ & $440.75 \pm 59.77$ & 0.271 \\
Sleep efficiency (\%) & $95.15 \pm 3.20$ & $95.22 \pm 3.31$ & $94.47 \pm 2.99$ & 0.302 \\
Sleep latency(min) & $3.30 \pm 2.31$ & $3.40 \pm 2.38$ & $3.14 \pm 2.10$ & 0.620 \\
Sleepiness score & $14.09 \pm 4.91$ & $14.17 \pm 4.95$ & $13.37 \pm 4.92$ & 0.001 \\
AHI & $43.88 \pm 20.66$ & $44.31 \pm 20.82$ & $37.68 \pm 21.06$ & 0.101 \\
Total time in sleep apnoea(min) & $160.18 \pm 95.90$ & $167.96 \pm 97.61$ & $124.25 \pm 83.16$ & 0.041 \\
REM (\%) & $16.79 \pm 6.50$ & $17.18 \pm 6.45$ & $16.19 \pm 6.62$ & 0.567 \\
Minimum Spo2 & $67.12 \pm 14.16$ & $66.64 \pm 14.20$ & $71.12 \pm 9.15$ & 0.138 \\
\hline
\end{tabular}

Data presented as mean \pm standard deviation. AHI: apnoea-hypopnea index; REM: rapid eye movement; and $\mathrm{SpO}_{2}$ : peripheral oxygen saturation (oximetry). 136 males and 24 females.

Table 2. Correlations between the excessive daytime sleepiness score and clinical variables, as well as between the excessive daytime sleepiness score and polysomnographic variables, in 160 patients with obstructive sleep apnoea hypopnea syndrome.

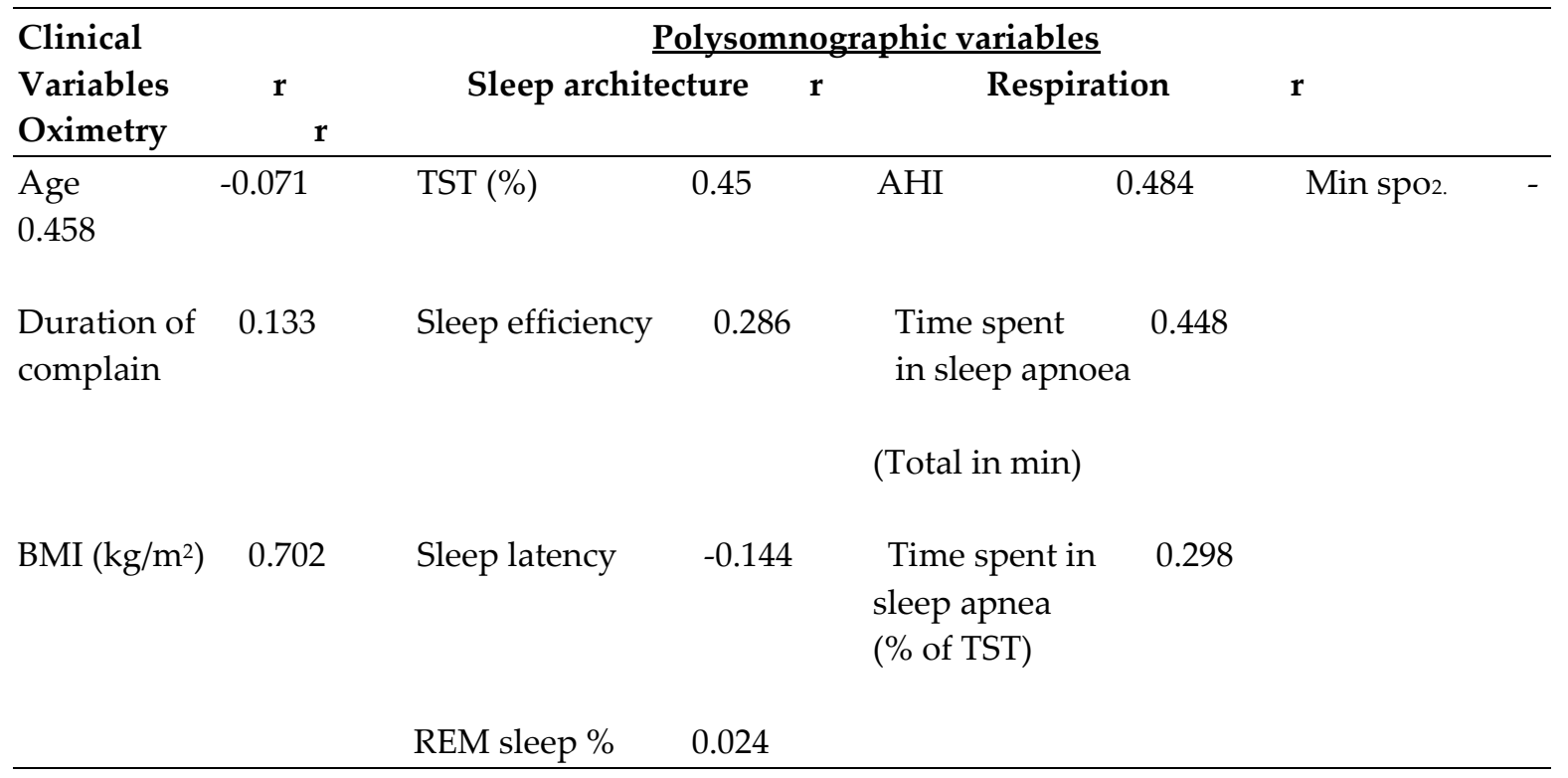

TST: Total sleep time; BMI: Body mass index; REM: rapid eye movement; AHI: apnoea-hypopnea index; $\mathrm{SpO}_{2}$ : peripheral oxygen saturation (oximetry); and Min: minimum. $\mathrm{P}<0.05$.

(C) 2020 by the authors. Submitted for possible open access publication under the terms and conditions of the Creative Commons Attribution (CC BY) license (http://creativecommons.org/licenses/by/4.0/). 